\title{
Las megauniversidades en el mundo
}

\author{
Olga Liliheh Matallana Kuan ${ }^{1}$ \\ Marleny Torres Zamudio ${ }^{2}$ \\ Leidy Johana Cárdenas ${ }^{3}$ \\ Cindy Viviana Rivera ${ }^{3}$ \\ Yohana Andrea Lache Palacios ${ }^{3}$ \\ Nydia Marilsen Riveros ${ }^{3}$
}

\begin{abstract}
Resumen
El artículo presenta una revisión de las tendencias educativas a nivel mundial, analizando la megauniversidad dentro del contexto de la globalización, a través de su definición y de la revisión general de diversas experiencias educativas de instituciones clasificadas como megauniversidades. Se parte del concepto de megauniversidad como una institución en donde la educación a distancia es, evidentemente, la actividad más importante y donde se han hecho inversiones en recursos tecnológicos de alta calidad y costo que permiten emplear la tecnología para propósitos educativos. Para su elaboración se conforma un grupo que indaga en variedad de artículos en la red y en información propia de las universidades en la web, construyendo una base de información amplia sobre su dinámica universitaria. Se encuentra que existen variables comunes entre ellas y que están correlacionadas con su crecimiento como megauniversidad, tales como su origen estatal para alcanzar objetivos de inclusión y cobertura, el uso necesario y vital de las tecnologías de la información y la comunicación (TIC) y la metodología de educación a distancia.
\end{abstract}

Palabras clave: megauniversidad, calidad, educación abierta, educación a distancia, metodología, mediaciones.

1 Economista, Especialista en Gerencia de Recursos Humanos. Master of Business Administration. Tutora UNAD CEAD, Duitama.

2 Economista, Especialista en Finanzas, Estudiante Master of Business Administration. Docente UNAD CEAD, Tunja. 


\begin{abstract}
The article presents a review of educational trends worldwide, analyzing the mega-university in the context of globalization, through its definition and general revision of various educational experiences in institutions classified as mega-universities. It starts from the concept of mega-university as an institution where distance education is obviously the most important and which have invested in high quality technological resources and cost permitting the use of technology for educational purposes. In its work-out, a group investigates variety of articles on the net and universities information on the web, building a comprehensive information on the dynamics of the university. We find that there are common variables between them and correlated with their growth as a mega-university, such as your state origin to achieve goals of inclusion and coverage, the vital and necessary use of information and communication technologies (ICT) and methodology for distance education.
\end{abstract}

Key words: mega-university, quality, open education, distance education, methodology, mediations.

Recibido: 16 de septiembre de 2010

Aceptado: 19 de octubre de 2010

\title{
Introducción
}

La universidad a distancia es una de las alternativas educativas que ha permitido diversificar el concepto tradicional de enseñanza-aprendizaje. Ha presentado un rápido crecimiento, impulsado principalmente por el desarrollo tecnológico de las últimas décadas, facilitando la interacción tutor-estudiante y entre los mismos estudiantes, que aunque se encuentren en dimensiones temporales y espaciales distintas, pueden interactuar y desarrollar procesos académicos mucho más flexibles y heterogéneos.

Este proceso de continuo cambio ha hecho que se generen nuevas estrategias de políticas educativas, pues se cambia el paradigma de educación superior de élites a educación superior de masas, generando a nivel global nuevas y mejores alternativas educativas, pues se impone la democratización de la enseñanza y la necesidad de atender la demanda educativa cada vez más creciente. En este sentido, la Organización de las Naciones Unidas para la Educación, la Ciencia y la Cultura (Unesco, 2002) sostiene que: "El aprendizaje abierto y a distancia tiene 
el potencial de facilitar un acercamiento a la educación que esté más centrado en el alumno y más orientado al consumidor, lo que a su vez favorecería un vínculo más estrecho entre las instituciones educativas y las organizaciones, empresas e industrias de la comunidad local". Esto significa, además, que las instituciones universitarias deben transformarse y alternar sus actividades para convertirse en entes dinamizadores de procesos innovadores que fortalezcan la inserción de las comunidades locales en los entornos globales.

En este contexto, la figura de megauniversidad se ha convertido en una posibilidad de educación basada en el uso intensivo de la informática y la telemática como herramientas imprescindibles para lograr cobertura y acceso ya que, como se menciona en el informe sobre la educación superior en América Latina y el Caribe 2000-2005 de la Unesco (2007): "la globalización y la sociedad del saber constituyen dos grandes motores que están impulsando la masificación de la educación superior en América Latina, la cual se expresa tanto a través de los niveles de competencia en los mercados laborales como en la disposición de los hogares a sacrificar rentas y tiempo para capacitarse". Así, la democratización, el libre acceso y la demanda insatisfecha de educación superior perfilan a las megauniversidades como uno de los más importantes proveedores de educación a distancia a nivel mundial, que se enmarca muy bien dentro de los procesos de globalización y democratización de la educación.

Este artículo pretende analizar la megauniversidad dentro del contexto de la globalización a través de su definición y de la revisión general de diversas experiencias educativas de instituciones clasificadas como megauniversidades, según los trabajos de Daniel (1996) en su libro Mega-universities and Knowledge Media: Technology Strategies for Higher Education. Aunque la temática es interesante, hay cierto grado de dificultad para obtener datos detallados pues generalmente se recurre a la información de los websites, que no siempre permiten determinar con claridad algunas de las características relevantes de este fenómeno mundial. Finalmente, se concluye con algunas reflexiones sobre la importancia de la megauniversidad en los nuevos estándares educativos.

\section{La megauniversidad y el desarrollo de la tecnología}

Para Sir John Daniel, ex Presidente de la Open University y ex Subdirector General de Educación de la Unesco (1996), la megauniversidad se define como "una institución de educación a distancia con más de cien mil estudiantes en cursos activos, que combina tres criterios: educación a distancia, educación superior y tamaño".

Sostiene Daniel (1996) que el concepto solamente aplica a instituciones donde la educación a distancia es, evidentemente, la actividad más importante y donde 
se han hecho inversiones en recursos tecnológicos de alta calidad y costo que permitan emplear la tecnología para propósitos educativos. Adicionalmente, se caracterizan por lograr incrementar sustancialmente el acceso a la educación superior a un bajo costo mediante la expansión extraordinaria de la matrícula. Sin embargo, este autor advierte que la consolidación y desarrollo de este tipo de organizaciones requieren un enfoque más eficaz que el que se da en la enseñanza tradicional. Para Lampert (2000), las megauniversidades son agencias educativas importantes en el desarrollo de un país y de un continente y producen una revolución en la enseñanza y la educación, ya que llegan a un gran número de alumnos a la vez a un bajo costo, lo que sería imposible realizar mediante la educación convencional. La educación a distancia se ha considerado una salida capaz de disminuir las diferencias entre las clases sociales porque ofrece a todos indistintamente la oportunidad de estudiar, y al mismo tiempo al Estado la posibilidad de reducir el déficit fiscal, eliminando los gastos en educación. Este hecho implica necesariamente facilidades de acceso educativo y cobertura a nivel mundial para grandes poblaciones y en todos los niveles educativos, favoreciendo la gestión del conocimiento mediante el crecimiento del uso de internet y nuevas tecnologías educativas.

Daniel (1996) en su libro Mega-universities and Knowledge Media: Technology Strategies for Higher Education, señala que en 1994 había once megauniversidades a nivel mundial (ver tabla 1), pero en la conferencia del cincuentenario de la Universidad de Televisión de Shanghái llevada a cabo en mayo de 2010, mencionó que ya se podían contabilizar más de veinte, ${ }^{4}$ introduciendo además el término de hiperuniversidades empleado para instituciones de educación superior con más de un millón de estudiantes. Ejemplos de estas instituciones son la Universidad Abierta Allama Igbal de Islamabad, la Universidad Nacional Abierta Indira Gandhi en la India, la Universidad China de Televisión y la Universidad Anadolu de Turquía.

Tabla 1. Las megauniversidades según Daniel, $1996^{5}$

$\begin{array}{llll}\text { Country } & \text { Name of institution } & \text { Established } & \text { Abbreviation } \\ \text { China } & \begin{array}{l}\text { China TV Univessity } \\ \text { system }\end{array} & \text { CTVU } \\ \text { France } & \begin{array}{l}\text { Centre national } \\ \text { d' Enseignement a }\end{array} & & \\ & \text { Distance } & \text { CNED }\end{array}$

\footnotetext{
4 Se citan en esta conferencia universidades como Allama Iqbal Open University (AIOU) (Pakistán), Anadolu University (Turquía), Athabasca University, Canada's Open University (Canadá), Bangladesh Open University (Bangladesh), China Central Radio and TV University (China), Dr. B.R. Ambedkar Open University (India), Indira Gandhi National Open University (India), Modern University for the Humanities (Rusia), National Centre for Distance Learning (Francia), Payame Noor University (Irán), Sukhotai Thamathirat Open University (Tailandia), The Open University (UK), The University of South Africa (Suráfrica), Universidade Estácio de Sá (Brasil), Universitas Terbuka (Indonesia), University of Maryland University College (USA), Yashwantrao Chavan Maharashtra Open University (India).
} 


\begin{tabular}{|c|c|c|c|}
\hline Country & Name of institution & Established & Abbreviation \\
\hline India & $\begin{array}{l}\text { Indira Gandhi National } \\
\text { Open Univessity }\end{array}$ & 1985 & IGNOU \\
\hline Indonesia & Universitas Terbuka & 1984 & UT \\
\hline Iran & $\begin{array}{l}\text { Payame Noor } \\
\text { University }\end{array}$ & 1987 & PNU \\
\hline Korea & $\begin{array}{l}\text { Korea National Open } \\
\text { Univesity }\end{array}$ & 1982 & KNOU \\
\hline South Africa & $\begin{array}{l}\text { University of South } \\
\text { Africa }\end{array}$ & 1873 & UNISA \\
\hline Spain & $\begin{array}{l}\text { Uiversidad Nacional de } \\
\text { Educacióin a Distancia }\end{array}$ & 1972 & UNED \\
\hline Thailand & $\begin{array}{l}\text { Sukhothai } \\
\text { Thammathirat Open } \\
\text { University }\end{array}$ & 1978 & STOU \\
\hline turkey & Anadolu University & 1982 & $A U$ \\
\hline United kingdom & The Open University & 1969 & UKOU \\
\hline $\begin{array}{l}\text { Notes } \\
\text { 1. As the Korea } \\
\text { 2. As the Univer }\end{array}$ & $\begin{array}{l}\text { orrespondence university } \\
\text { Cape of Good Hope }\end{array}$ & & \\
\hline
\end{tabular}

En términos generales, las megauniversidades requieren sistemas de gestión complejos y una especial atención al nivel de calidad e innovación tecnológica y pedagógica de sus contenidos académicos. La gran mayoría de instituciones analizadas cuentan con sistemas duales que combinan la virtualidad y otras mediaciones pedagógicas para lograr interacción directa con los estudiantes. El gran reto consiste en generar, más que aprendizajes, competencias que sean útiles en un entorno globalizado y de sistemas transnacionales que obliga a desarrollar políticas educativas integrales que garanticen calidad y expansión de operaciones educativas y económicas fuera de las fronteras de un país.

\section{Características de algunas megauniversidades}

Asia

Para Daniel, citado por Silvio (1998), las megauniversidades han proliferado principalmente en países asiáticos pues logran combinar alta cobertura y equidad de acceso con la calidad académica. En Asia se encuentran siete de las once megauniversidades estudiadas por Daniel; es lógico que así sea, pues allí se concentra la mayor parte de la población mundial y sus países confrontan serios problemas de acceso al sistema educativo superior.

Según estudios de la Unesco (2005), China está haciendo un gran esfuerzo en el desarrollo de la educación a distancia basada en las TIC y el e-learning como una 
forma de satisfacer la creciente demanda de educación superior y la necesidad de personal calificado en una economía en rápida expansión y emergente. En China, la educación superior es gratuita y, en lo que respecta la educación a distancia, es supervisada y coordinada por el gobierno mediante el ministerio de educación; existe gran variedad de universidades abiertas, varios colegios de educación a distancia, escuelas y departamentos que se encuentran en universidades y colegios convencionales. ${ }^{6}$

La megauniversidad más representativa de China es la Centre Radio and Televisión University of China CRTVU, de acuerdo con las investigaciones de Luzzi (2007) y los datos encontrados en el website. La CRTVU, situada en Beijing, fue creada en 1979, es una institución que depende del ministerio de educación nacional con estatus de universidad nacional, tiene representación en 44 provincias donde existen sistemas de radio y televisión de la universidad denominados RTUV, consta de 841 escuelas a nivel municipal y de 1780 estaciones que operan en los niveles local, regional y nacional. Hay diversas instituciones como la universidad de educación de adultos, el departamento de educación por correspondencia, el instituto de educación en línea, que buscan satisfacer necesidades educativas de distintos sectores de la población.

La universidad tiene como misión principal el desarrollo de cursos de educación superior para los profesionales, los trabajadores de las empresas, el personal de las fuerzas armadas y otros miembros de distintas clases sociales que ven en la educación superior una oportunidad de crecimiento. Los estudiantes aspirantes a ingresar a la CRTVU a nivel de pregrado deben ser ciudadanos chinos y haber aprobado el examen de ingreso; sin embargo, quienes aplican para mejorar sus competencias profesionales no requieren este examen de ingreso.

De acuerdo con los datos aportados en la página institucional de la universidad, ${ }^{7}$ se ofrecen más de 580 cursos en áreas del conocimiento como agricultura, ingeniería, medicina, literatura, derecho, economía, negocios, educación e historia. Estos cursos no solamente se orientan a desarrollar contenidos técnicos sino que además se fundamentan en la ideología socialista. Las mediaciones se realizan a través de radio, televisión, medios impresos, materiales visuales y redes informáticas, lo que permite llegar a zonas rurales que puedan tener dificultades en el manejo de internet. Este crecimiento en cobertura también ha posibilitado un fuerte incremento de estudiantes matriculados, de 97.502 en 1979 a 2.3 millones en 2004, presentando un vertiginoso aumento en un lapso relativamente corto.

Según lo menciona Jung en sus estudios acerca de la calidad de estas instituciones (2005:86), las áreas de control de calidad claves en la educación a distancia de 
esta megauniversidad son: política y planificación, diseño y desarrollo del curso, servicios de apoyo a los estudiantes, evaluación de aprendices, tecnología y medios de comunicación, y requisitos unificados.

En la India, la Indira Gandhi National Open University (IGNOU) fue creada por el Parlamento indio en $1985^{8}$ y comenzó sus actividades en 1987. Su misión es avanzar y diseminar el aprendizaje y el conocimiento por una diversidad de medios y tecnologías de comunicación. Sus objetivos principales son: proveer de oportunidades de educación a vastos segmentos de la población, incluyendo los grupos desfavorecidos; promover el concepto del aprendizaje a distancia y suministrar educación de alta calidad a nivel universitario (Silvio, 2000:236).

Esta universidad ha mantenido una tendencia constante de crecimiento, pues pasó de 4.528 alumnos en 1987 a 91.398 en 1995 y a 492.542 en 2006. Sin embargo, la institución no tiene tasas de retención satisfactorias pues según estudios de Fozdar, Kumar y Kannan (2006), la tasa de deserción del primero al segundo año es de $51,7 \%$ en promedio, y de éstos sólo el $73 \%$ aproximadamente estudia el tercer año, lo que significa que un gran número de estudiantes no concluye sus estudios.

En lo que respecta a la oferta educativa hay programas de pregrado, maestría y doctorado, servicios de extensión universitaria dirigidos a la formación profesional, y las áreas de conocimiento son: Informática, Educación, Ciencias de la Salud, Humanidades, Administración y Ciencias. El número de cursos ha crecido paralelamente al número de programas: en 2004 había 88 programas y 820 cursos. La universidad ha empleado el modelo multimedia, programas de radio, website y la tutoría interactiva por audio y video, agregando actividades de campo, experiencias prácticas y laboratorios.

La IGNOU trabaja con material impreso, que es preparado por un equipo de especialistas a nivel nacional de forma muy estricta. Los videos se transmiten por televisión educativa a ciertas horas. Respecto a la radio, hay 186 estaciones en todo el país que trabajan principalmente los domingos, invitando a expertos en diversos temas para complementar la información; estos expertos pueden contestar llamadas hechas a números telefónicos gratuitos en más de ochenta ciudades y en tiempo real. Hay también un repositorio de objetos virtuales destinados permanentemente para el uso de los estudiantes, una red de 1.400 centros de estudio y prácticas de laboratorio para estudiantes de diferentes partes del país.

Las áreas claves de control de calidad para esta megauniversidad, según Jung (2005:86), son: necesidades y objetivos del programa, contenido y nivel del programa, duración del programa, presentación del contenido, 
transformación del contenido para ser empleado en educación a distancia y evaluación de los estudiantes.

De acuerdo con Silvio (2000:237), otra megauniversidad es la Korea National Open University (KNOU), con sede en Seúl, surgida de la antigua Korea Air and Correspondence University (KACU) establecida en 1972. En 1994, ésta cambió su nombre por el actual. Los objetivos de la KNOU son: elevar el nivel educativo de la población brindando oportunidades de estudio a través de métodos de educación a distancia a graduados de educación secundaria, quienes por diversas razones no pueden estudiar o han interrumpido sus estudios universitarios; mejorar la suficiencia académica y tecnológica de la gente ya comprometida en una profesión, ofreciéndole oportunidades para continuar sus estudios en las áreas principales de la ciencia y la tecnología modernas y, por último, contribuir al bienestar de la nación y la sociedad. ${ }^{9}$

La universidad cuenta con cuatro facultades que ofrecen títulos en lengua y literatura, derecho, administración pública, economía, administración, comercio internacional, turismo, medios de comunicación, agricultura, economía doméstica, salud ambiental, enfermería, educación infantil y cultura. Posee una sede principal, catorce sedes regionales y 35 centros pequeños de estudio distribuidos en todo el país.

La KNOU emplea diferentes medios de comunicación: radio y televisión abierta, televisión por cable, vídeo y audio, videoconferencia, CD-ROM, Internet y acceso a redes informáticas. El sistema de videoconferencia se conecta a trece centros de estudios regionales. Este sistema proporciona un entorno educativo similar al estilo del aula (presencial) donde los estudiantes pueden participar activamente, asistiendo a conferencias y recibiendo orientación de manera inmediata.

Respecto al número de estudiantes, según datos de Jung (2005:245), la universidad contaba con 196 mil pero en 2000 ya tenía 350 mil. También presenta un alto nivel de deserción académica, lo que ha hecho que la universidad pretenda ser de vanguardia, basada en cinco estrategias, que de acuerdo con Jung (2000: 49) son: 1) base de datos en línea y biblioteca digital multimedia, que incluye material de base y complementario para más de trescientos cursos y un canal oficial de televisión y radio, para la interacción de tutores y estudiantes; 2) conexión nacional a través de videoconferencia, que ha permitido la conexión de catorce centros diferentes y la interacción de estudiantes y tutores empleando la línea comercial T1 (portadora digital disponible para grandes volúmenes de voz ,tráfico de datos y video comprimido); 3) canal de TV por cable y satélite educativo para proporcionar servicios a los estudiantes que viven en regiones remotas, lo que permite que la universidad llegue a cerca de nueve millones de personas en el país sin incrementar demasiado los costos de 
transmisión; 4) asociación con ocho universidades, en un consorcio conocido como la Universidad Virtual de Korea (KVU), lo cual ha proporcionado una alternativa a la educación superior y capacitación laboral para los trabajadores adultos (en la experiencia de Corea se puede observar que las alianzas han sido de una enorme importancia para el desarrollo de sistemas con costos reducidos, permitiendo al mismo tiempo un mayor avance tecnológico y una mejor calidad en el desarrollo de programas de estudio); 5) sistema integrado de web para mejorar los servicios a estudiantes, de gran eficacia administrativa en registro de los estudiantes, programas de estudio, reserva de libros en biblioteca, etc.

Según Jung (2005:87), las áreas clave de control de calidad en esta institución son: servicios de apoyo a los estudiantes, tutorías, e-learning, desarrollo de libros de texto y difusión de programas en desarrollo.

La Payame Noor University (PNU), de Irán, fue creada en 1987 especialmente como única universidad abierta y a distancia del país. Es considerada, según Silvio (2000:238), como la universidad menos costosa, más efectiva y de mayor cobertura del país. Es una universidad estatal coordinada por el ministerio de ciencia, investigación y tecnología de Irán. De acuerdo con las investigaciones de Luzzi (2007: 243), su principal objetivo es democratizar las posibilidades de acceso a la educación para satisfacer las necesidades vitales para el desarrollo de Irán.

Cuenta con 257 centros, emplea mediaciones como folletos de instrucción, cintas de vídeo, programas educativos en televisión, películas, website, además de tutorías los fines de semana. Ofrece más de setenta programas de pregrado, postgrado y maestría, licenciaturas, ingeniería, arquitectura, química, ingeniería informática, tecnologías de la información, ciencia s de la computación, educación, geología, biología, psicología, etc. ${ }^{10}$

Esta institución contaba con ocho mil estudiantes activos en 1987 y en 2004 contabilizaba 467.000 ( Luzzi 2007:385). Cuenta además con un interesante sistema de estímulos a los mejores egresados de postgrado, consistente en que si están desempleados y obtuvieron buenas calificaciones académicas, se les da una oportunidad de empleo dentro de los programas de la universidad.

La Universitas Terbuka (UT) de Indonesia fue creada por el gobierno en 1984 en la ciudad de Yakarta y se ha convertido también en la megauniversidad a distancia de ese país. La universidad no sólo es responsable de expandir las oportunidades educacionales sino al mismo tiempo de fortalecer el compromiso del gobierno de mejorar la calidad de la educación y hacerla más pertinente a las necesidades nacionales. Además provee oportunidades educativas a personas 
que por diversas razones no pueden asistir a centros educativos superiores convencionales y tradicionales (Silvio, 2000:239).

De acuerdo con las investigaciones de Luzzi (2007: 219), su objetivo principal es ampliar las oportunidades educativas en el territorio nacional mediante una educación más orientada a las necesidades del país. La admisión a la universidad es flexible, pero se requiere como mínimo estudios de secundaria completa. En 1984, la universidad tenía 84 mil estudiantes; en 1998, ascendió a 442 mil; en 2003 se redujo a 220 mil. Este hecho se atribuye, según investigaciones de Pannen (2003:4), a la crisis económica que desde 1998 ha abatido al país, y a que un alto número de estudiantes eran profesores que culminaron sus estudios de postgrado.

Luzzi (2007: 220) menciona que la UT tiene un servicio en línea integrado para los estudiantes que permite el apoyo de información académica general (programas y planes de estudios, notas, anuncios de exámenes, creación de auto-exámenes, horarios de tutoría en la radio), además de información académico-administrativa (registro, transferencia de crédito, certificados), tutoriales en línea (basado en la infraestructura de la red), así como cursos de la infraestructura básica de la web.

La UT ofrece 28 programas organizados en 1200 cursos; respecto a los materiales de estudio y la estrategia educativa se emplean reuniones cara a cara, programas de televisión y radio, módulos, servicios postales, servicios de fax o programas pregrabados. Para facilitar el acceso a estudiantes que viven en zonas muy apartadas, cuenta también con simuladores de laboratorio y laboratorios móviles equipados para llevar a cabo experimentos científicos, incluyendo una computadora conectada a Internet.

El desarrollo progresivo de las tecnologías de la información y la comunicación en Indonesia ha abierto un nuevo campo oportunidades para la UT; sin embargo, el país cuenta aún con un bajo número de usuarios en internet y una escasa penetración de computadora portátil que fluctúa alrededor del $5 \%$, por lo que se impone el uso de metodologías diversas para lograr los cometidos de acceso educativo.

La Anadolu Üniversitesi es una universidad pública situada en Eskisehir, Turquía, creada en 1982 de la unión de cuatro instituciones de educación. La mayor parte de las facultades y las escuelas, incluyendo la facultad abierta de educación, están situadas en el campus de Yunus Emre en Eskisehir. Para servir a sus estudiantes de educación a distancia, la universidad de Anadolu funciona con 88 centros administrativos u oficinas en áreas urbanas a través de Turquía. La meta de la universidad es educar a los turcos que viven en áreas rurales y a otras personas que no cuentan con los recursos para ingresar en universidades convencionales. La inscripción en programas abiertos de educación ha aumentado de treinta mil 
en 1982 a 870 mil en 2005. La universidad de Anadolu cuenta con programas de educación a distancia en doce facultades, seis colegios, nueve escuelas de formación profesional y 23 institutos de investigación, contando en todos estos programas con más de un millón de estudiantes. ${ }^{11}$

Las mediaciones empleadas incluyen difusiones de radio, videoconferencias e Internet. Los estudiantes pueden también tener acceso al asesoramiento académico o clases opcionales en algunas de las oficinas de la universidad situadas a lo largo de Turquía. ${ }^{12}$

Según Aydin y Ulutak, el 30\% de los alumnos actuales de educación a distancia viven en zonas rurales donde tienen dificultades para acceder a computadoras e Internet; por eso, la mayoría de estos programas siguen basados en correspondencia y el número de alumnos en línea es sólo el $2 \%$. El éxito en la mayoría de los programas está determinado por múltiples pruebas de rendimiento que permiten a los estudiantes evaluar su desempeño.

La Universidad Abierta Sukhothai Thammathirat (STOU), según Luzzi (2007:244), fue fundada en 1978 como universidad estatal, convirtiéndose en la decimoprimera universidad estatal y la primera universidad abierta del sudeste asiático. La STOU se creó con el propósito de ampliar las oportunidades para la educación superior de adultos, contribuir al desarrollo del país y preservar la cultura e identidad de la nación tailandesa. En el año 2004 había aproximadamente 173 mil estudiantes registrados. Las mediaciones empleadas en esta institución incluyen programas de televisión, boletín de noticias en radio, información a través de su web oficial y un centro de información telefónica para responder a las preguntas de los estudiantes o público en general.

La STOU ofrece títulos de pregrado, postgrado, másteres y cursos cortos. La universidad ofrece materiales educativos que envía a cada alumno a través de medios electrónicos; se utilizan diversos materiales multimedia, agendas de trabajo, tutorías, cintas, videos, televisión, radio, e información y resultados de las pruebas. La universidad aplica las pruebas en todas las provincias del país para facilitar el acceso de sus recursos a los estudiantes. Las pruebas se aplican dos veces por semestre, incluyendo una revisión periódica y un examen final. Los resultados se envían a los estudiantes a través de correo electrónico de 30 a 45 días después, dice Luzzi (2007:246).

\section{África}

Ésta es la segunda región del mundo por su tamaño, y una de las más diversas a nivel cultural y lingüístico; gran parte de la población pobre del mundo vive en 
este continente que se enfrenta a un rápido crecimiento y a la urbanización de su población que demanda mayores oportunidades educativas.

La Universidad de Sudáfrica, UNISA, es una megauniversidad que sirve a más de cien mil estudiantes. Si bien es aún una universidad a distancia que se vale todavía de medios de comunicación tradicionales, está proyectando expandirse al ciberespacio para complementar esos medios con los electrónicos. (Silvio 2000:239).

De acuerdo con las investigaciones de Luzzi (2007:252), la UNISA es considerada la universidad más antigua en el sur de África, fue fundada en 1873 como Universidad de Cabo de Buena Esperanza y pasa en 1946 a ser universidad a distancia. Sus objetivos son participar en el desarrollo de la comunidad local con sus recursos potenciales para el desarrollo de la población de bajos ingresos. Según los datos del estudio de Luzzi (2007: 385), la universidad pasó de 130 mil estudiantes matriculados en 1995 a 226.424 en 2004.

Esta universidad ha desarrollado un portal para apoyar actividades de comunicación entre profesores, estudiantes y la institución, donde se puede acceder a servicios de resultados de pruebas, financiación, material educativo, boletín de noticias, directorio de puestos de trabajo y becas de estudios.

La UNISA cuenta con facultades de economía, humanidades, derecho, agricultura y ciencias del medio ambiente, tecnología y nueve centros donde los estudiantes reciben tutorías y cuentan con servicios de laboratorios de computación, bibliotecas y servicios de información. El sistema educativo es flexible porque los estudiantes no tienen que realizar un examen nacional de admisión. Una vez que se inscriben, reciben guías de estudio que contienen materiales necesarios para clase. Durante el curso deben realizar varias actividades y enviarlas a los tutores de sus centros de estudio. La universidad cuenta con tutorías presenciales y para los estudiantes que viven en zonas aisladas, hay sistema de videoconferencia.

En cuanto a la evaluación de la educación, la UNISA tiene una estructura compleja que se centra en la administración de exámenes, ya que, de acuerdo con la investigación de Luzzi (2007:252), tiene muchos centros acreditados repartidos en todos los continentes del mundo. El código disciplinario de los estudiantes es estricto; éstos pueden entrar al centro de exámenes más cercano a su residencia; las pruebas prácticas para estudiantes de artes visuales y multimedia deben incluir trabajos y declaraciones juradas que contengan fotos firmadas que muestran el proceso de creación y el lugar en el que se desarrolló el trabajo.

\section{Europa}

La Universidad abierta del Reino Unido, UKOU, inició oficialmente sus actividades en 1971 con 25 mil estudiantes y en 2004 contaba con 220 mil matriculados. Su sede 
principal se encuentra ubicada en Walton Hall y cuenta con trece sedes regionales. Como lo menciona Silvio ( 2000:227), ha sido una institución considerada un modelo de inspiración de la educación a distancia en todo el mundo, pues ha empleado progresivamente todos los medios de comunicación para la enseñanza y los ha integrado de manera coherente, desarrollando con éxito cursos en CDROM, conferencias por computadora, tutorías mediante videoconferencias y correo electrónico; ha combinado el desarrollo de su página web con transmisiones televisivas y el empleo de tecnología para la administración. Se destaca en los estudios de Daniel (2001:338) el compromiso institucional respecto a los procesos de investigación, lo cual se explica en parte porque la Open University forma parte de la élite de las universidades británicas donde la mayoría de programas están clasificados como excelentes.

Según lo describe Daniel (1997), la Universidad Abierta del Reino Unido cuenta con estudiantes, en su mayoría adultos, que trabajan y efectúan estudios de tiempo parcial, mientras que en las universidades chinas por TV la mayor parte son adultos que perciben remuneración para cursar estudios de tiempo completo. Daniel (2001) caracteriza a la Open University como generadora de materiales multimedia de alta calidad, con apoyo académico asignado de cada facultad para cada veinte estudiantes, con una buena logística y un alto porcentaje de investigadores.

Para Luzzi (2007: 268) es destacable la metodología de enseñanza y aprendizaje, pues cuenta con gran cantidad de material multimedia producido en asociación con la BBC de Londres, lo que implica gran calidad de los materiales. Cuenta también con material para estudiantes con necesidades especiales, y con materiales de acceso libre que pueden ser descargados y modificados por educadores de acuerdo a sus necesidades, siempre que se cumplan determinadas normas de licencia establecidas para su uso.

Según Jung (2005:86), la UKOU presta mucha atención a los aspectos de calidad, cuyas áreas claves son: guía de normas para la calidad, que cubre ocho hojas de datos, gestión institucional de calidad y normas, marco para la calidad y las normas académicas, examen interno, evaluación y premios de colaboración; apoyo y orientación al estudiante, staff de personal y rendición de cuentas a las partes interesadas.

El Centro Nacional de Educación a Distancia (Centre National d'Enseignement à Distance) (CNED) de Francia, según Silvio (2000: 228), abarca todos los niveles de enseñanza, es un establecimiento público del ministerio de educación nacional de Francia creado en 1939. Tiene siete antenas nacionales en todo el territorio francés y nueve internacionales en diversos territorios franceses de ultramar. Es un ejemplo de integración y articulación entre los distintos niveles de enseñanza a través de nuevas modalidades de educación. Ofrece actualmente cerca de tres mil cursos en todos los niveles de enseñanza, incluyendo la formación profesional. 
Según las investigaciones de Luzzi (2007: 279), esta universidad cuenta con amplia experiencia en los métodos de enseñanza y conocimientos técnicos, para el desarrollo de proyectos de educación a distancia. En 1995 había 184.614 estudiantes y en 2000 ascendió a 368.163.

Según Jung (2005:86), las áreas claves de control de calidad en esta institución son: política y planificación, provisión y desarrollo de recursos humanos, gestión y administración de estudiantes, diseño y desarrollo de programas, diseño y desarrollo de cursos, apoyo a los estudiantes, evaluación de aprendices y medios de comunicación para la enseñanza.

En general, las organizaciones analizadas no sólo se caracterizan por su enorme crecimiento en términos de número de estudiantes matriculados sino que también cuentan con características comunes como elevadas inversiones tecnológicas, empleo de diversas mediaciones y cuidado extremo en la elaboración de material didáctico que promueva la autogestión del conocimiento. Tal vez el gran reto de estas organizaciones es combatir los elevados niveles de deserción, incrementar la tasa de estudiantes efectivamente graduados y lograr estándares de calidad cada vez más exigentes, que realmente aseguren la educación para todos, y con altos niveles de cualificación.

\section{Conclusión}

La caracterización de estas megauniversidades permite inferir acerca de la importancia de nuevas tecnologías en el ámbito educativo moderno y del papel de la educación a distancia en el ámbito mundial. El conocimiento está al alcance de una gran cantidad de población que requiere de nuevas metodologías y mediaciones, para asegurar mayor inclusión y cobertura educativa.

Se espera la consolidación de instituciones de educación superior como megauniversidades, y frente a esta tendencia, el compromiso de generar nuevos medios y mediaciones tecnopedagógicas que respondan a las necesidades que ello demanda, como materiales de estudio, apoyo académico, logística y conformación de redes de conocimiento que propicien ambientes favorables para la investigación y el desarrollo de las comunidades.

El atractivo de las megauniversidades radica en que buscan atender necesidades variadas de una población estudiantil por demás heterogénea, que dado el desarrollo masivo de Internet, cuenta con un sinnúmero de oportunidades de innovación académica, pedagógica y didáctica que le permiten su inserción en el mundo educativo. 
Así, en estas instituciones se da la posibilidad de conformar redes institucionales a través de alianzas estratégicas considerables que permiten alcanzar los beneficios de las economías de escala.

\section{Referencias bibliográficas}

Aydin, Hakan, Y Ulutak, Nazmi. Open Education Resources of Anadolu University, Turkey en www.eprints.knust.edu.gh/182/1/Aydin.pdf.

Daniel, John. (1996). Mega-universities and Knowledge Media: Technology Strategies for Higher Education, London.

(1997). La mega universidad: ¿La academia para el nuevo milenio?.Revista de educación superior en línea No 116, Extraído en julio 26 de 2010 de http://www.anuies. $\mathrm{mx} / \mathrm{servicios/p \_ anuies/publicaciones/revsup/res116/txt7.htm.}$

. (2001). El surgimiento de la mega universidad, en La visión de los líderes en la era digital, Anne Leer -editora-, Pearson Educación, México. p. 338.

Fozdar, Bharatinder; Kummar, Lalita S.; Kannan, S. A survey of a study on the reasons responsible for student dropout from the bachelor of science programme at Indira Gandhi National Open University. India, 2006. .International Review of Research in Open and Distance Learning. vol. 7,n.3. Diciembre, 2006, Extraido en septiembre 18 de 2010 de http://www.irrodl.org/index.php/irrodl/article/download/291/755.

Jung, Insun. (2000). Korea: Can Edutopia Become a Reality? En http://www. techknowlogia. org/TKL_Articles/PDF/99. pdf extraído el 27 de agosto de 2010, p. 49.

(2005). Quality assurance survey of mega universities. Extraído el 27 de julio de 2010 de www.col.org/.../pseries_llldhe_cho7.pdf, p. 86.

Lampert, Eliani. (2000). Educación a Distancia ¿Elitización o alternativa para democratizar la enseñanza?, Revista Perfiles Educativos No.88, Universidad Nacional Autónoma de México, p. 3.

Luzzi. (2007). O papel da educação a distancia na mudança de paradigma educativo: da visão dicotômica ao continuum educativo, Tesis de Doctorado para la obtención del título de Doctor en Educación, Facultad de Educación de la Universidad de São Paulo, Brasil. Área de Concentração: Educação - Opção: Estado, Sociedad de Educação, Orientador: Prof. Dr. Pedro Roberto Jacobi.

Pannen, Paulina PhD. (2003). Distancia de las políticas públicas de educación y la práctica en la educación superior: el caso de Indonesia. en http://www.abed.org. 
br/revistacientifica/ Revista_PDF_Doc/2003_Distance_Education_Public_Policy_ Paulina_Pannen.pdfhttp:// www.abed.org.br/revistacientifica/Revista_PDF_ Doc/2003_Distance_Education_Public_Policy_Paulina_Pannen.pdf.

Silvio, José (1998). La virtualización de la educación superior: alcances, posibilidades y limitaciones. Revista Educación Superior y sociedad, Volumen 9, (27-50).

.......... (2000). La virtualización de la universidad: ¿cómo transformar la educación superior con la tecnología? José Silvio, leslac, Caracas.

Unesco (2002). Aprendizaje abierto y a distancia. Consideraciones sobre tendencias, políticas y estrategias. Patru, Mariana (editor), ediciones Trilce, Montevideo, Uruguay, p. 44.

(2005). Perspectives on distance education: lifelong learning and distance higher education. Christopher McIntosh, Editor Zeynep Varoglu, Editorial Coordinator.

. (2007). Informe sobre la educación superior en América latina y el Caribe 20002005. La metamorfosis de la educación superior, Caracas, p. 15. 